\title{
基于数字孪生模型的直驱部件高精度控制方法
}

\author{
江献良 陈凌宇 郑杰基 谭若愚 李宝宇 范大鹏 \\ (国防科技大学智能科学学院 长沙 410073)
}

\begin{abstract}
摘要: 直驱部件以结构紧凑、失动量小和无间隙传动的优势在高档数控机床、航空航天精密伺服机构等重大装备中得到了广 泛应用。直驱部件的伺服控制精度和动态响应特性是伺服系统的重要指标, 针对负载惯量摄动和装配特性的变化造成系统伺 服性能的下降甚至失稳的现象, 提出一种基于数字孪生模型的复合控制方法, 以物理实体数据驱动数字孪生模型参数同步, 以数字孪生模型优化物理实体的伺服控制参数。首先提取了直驱系统的关键特征参数, 用集总参数模型描述线性环节, 用非 线性参数模型描述非线性特征, 建立了直驱部件的虚拟物理模型; 采集物理实体状态数据, 提出了基于递推增广最小二乘算 法的数字孪生模型参数更新方法, 实现了模型特征的自适应同步; 利用同步参数优化系统控制参数, 提出了指标约束下的速 度控制器和扩张状态卡尔曼滤波器设计方法, 形成了数字孪生驱动的复合控制策略。实验表明, 提出的方法具有更好的抵抗 惯量摄动能力, 模型同步算法能准确快速地辨识直驱系统的惯量变化; 相对传统方法, 跟随 $20 \% / \mathrm{s} \& 1 \mathrm{~Hz}$ 正弦信号的速度残差 均方根由 $5.88 \%$ 减少为 $1.76 \%$, 优化了 $70 \%$; 阶跃响应过程的调整时间始终满足设计指标 $0.1 \mathrm{~s}$, 实际响应曲线与理论响应 曲线的 Pearson 相关系数由 0.957 提高到了 0.993 。研究成果为进一步细化数字孪生模型颗粒度和推进数字孪生模型在运动控 制中的应用提供参考。
\end{abstract}

关键词: 数字孪生模型; 惯量摄动; 状态观测器; 参数同步方法; 复合控制

中图分类号: TP202

\section{High-precision Control Method of Direct Drive Components Based on Digital Twin Model}

\author{
JIANG Xianliang CHEN Lingyu ZHENG Jieji TAN Ruoyu LI Baoyu FAN Dapeng
}

(College of Intelligence Science and Technology, National University of Defense Technology, Changsha 410073)

\begin{abstract}
With the advantages of compact structure, small loss of momentum and no gap transmission, direct drive components are widely used in high-end CNC machine tools, aerospace precision servo mechanisms and other major equipment. The dynamic response characteristics and robust performance of direct drive components are important indexes of the servo system. However, the perturbation of load inertia and changes in assembly characteristics will cause the system's servo performance to decrease or even lose stability. In order to improve the system's servo performance and motion safety, A composite control method based on digital twin model is proposed, which uses physical entity data to drive virtual model parameter synchronization, and uses virtual model to optimize physical entity servo parameters. Firstly, the key characteristic parameters of the direct drive system are extracted, the linear link is described by the lumped parameter model, the nonlinear feature is described by the distributed parameter model. Then, the virtual physical model of the direct drive component is established. The parameter updating method of the digital twin model based on the recursive augmented least squares algorithm is proposed to realize the adaptive synchronization of the model features. The control parameters of the system are optimized by using synchronous parameters. The design method of the speed controller under index constraints and the extended state Kalman filter is proposed to form the compound control strategy. Experiments show that the method proposed has better resistance to inertia perturbation, and the virtual model parameters can identify the inertia change of the direct drive system accurately and quickly; the root mean square of the velocity residual following the $20^{\circ} / \mathrm{s} \& 1 \mathrm{~Hz}$ sinusoidal reference signal is reduced from $5.88 \%$ s to $1.76 \%$, which is optimized by $70 \%$; the adjustment time of the step response process always meets the design index, the Pearson correlation coefficient of the actual response curve and the theoretical response curve has been increased from 0.957 to 0.993 . The research result is to further refine the digital twin model and promote the application of
\end{abstract}

* 自然科学基金委与湖南省区域创新发展联合基金(U19A2072)资助项目。

20210112 收到初稿, 20210419 收到修改稿 
digital twin model in motion control.

Key words: digital twin; inertia perturbation; state observer; parameter synchronization method; compound control

\section{0 前言}

随着数控机床、工业机器人、精密指向装置、 自动化生产线、直线运动装置等智能制造装备应用 领域和功能的不断丰富, 力矩电机直驱系统需要执 行越来越复杂的运动任务, 也面临着惯量摄动和装 配特性变化等各种模型时变问题。

在机器人不同姿态下耦合惯量、数控机床切削 深度变化、机械臂抓取不同重物以及精密指向装置 载荷变化等常见工况中, 负载惯量摄动和摩擦特性 的变化成为了影响直驱部件动态性能的关键因素。 针对直驱部件在惯量摄动和摩擦特性变化情况下的 高精度控制问题, 许多学者进行了相关的研究。

传统的比例积分控制器凭借参数少和调试简单 的优点广泛应用于工程实践中 ${ }^{[1]}$, 但是恒增益控制 器的鲁棒性不足以适应不确定系统的参数变化, 难 以克服摩擦导致的爬行现象 ${ }^{[2]}$ 。李国涛等利用自适 应鲁棒控制器解决抓取机构的参数不确定问题, 但 是鲁棒控制器在实际应用中偏于保守, 不利于发挥 运动控制部件的最优性能 ${ }^{[3]}$ 。除了传统方法外, 许 多学者探索了神经网络、机器学习和遗传算法等智 能算法 ${ }^{[4-6]}$, 智能算法虽然具有建模简单、寻优能力 强等优点, 但是这些算法计算负荷大, 收玫时间长, 参数大多不具备实际物理意义, 在应用中受到了一 些限制。

数字孪生是在虚拟系统中建立实际物理系统的 数字映像, 通过数据交互、状态同步和迭代优化等 手段, 为物理实体的规划设计、状态监控和运动控 制扩展新的能力。自 2003 年美国密歇根大学 GRIEVES 教授首次提出以来, 数字孪生技术被逐渐 推广到了运动控制领域。美国国防部将数字孪生技 术用于飞行器的健康维护与保障。陶飞等 ${ }^{[7]}$ 提出了 包含物理实体、虚拟实体、连接、孪生数据以及服 务的数字孪生五维模型。华中数控采集运动轴状态 数据形成机床的数字孪生模型, 实现主轴电流的在 线检测及其工艺优化, 提高了加工效率 ${ }^{[8]}$ 。奥克兰 大学学者建立数控机床加工过程的信息物理模型, 形成包含加工工具信息模型的信息物理生产系统, 服务于机床安全状态监测过程 ${ }^{[9]}$ 。

通过将运动学和动力学信息体现到数字孪生 模型中, 数字孪生驱动的伺服系统越来越具备实时 数据分析和自主功能, 因而为解决运动部件在惯量
摄动和摩擦变化下的高精度控制问题提出了新的 解决思路。庞宇等建立了行星齿轮箱数字孪生体动 力学模型, 高度模拟真实设备的振动状态 ${ }^{[10]}$ 。PI 等将机电耦合模型用于研究高速加工中的轮廓误 差估计和补偿技术 ${ }^{[11]}$ 。RIDWAN 等通过数字孪生 模型中的实时数据反馈, 实现了进给速度的自适应 优化 ${ }^{[12]}$ 。TONG 等建立了包含伺服控路、机械运动 和动力学状态的铣削过程数字孪生模型, 定位了轴 系运动波动的原因并通过调节伺服参数消除了误 差 $^{[13]}$ 。总的来说, 数字孪生模型在运动控制领域的 应用仍然面临许多困难 ${ }^{[14]}$, 主要分为两个方面: 一 是多维度物理模型构建问题, 另一个是模型参数同 步问题。物理实体与数字孪生模型之间的实时一致 性是构建数字孪生模型的基础, 数字孪生模型可用 于监控物理实体, 而物理实体可发送数据以更新其 数字孪生模型, 进而实现对物理模型的数据管理、 状态更新、预测控制和安全监控等 “服务” 功 能 $^{[15-16]}$ 。

本文针对直驱部件在惯量摄动下的高精度伺服 控制问题, 提出了一种基于数字孪生系统的解决方 案。通过采集物理实体状态数据, 实现直驱系统数 字孪生模型的状态更新和参数同步, 利用模型参数 优化调整基于性能指标约束的速度控制器和扩张状 态卡尔曼滤波器参数, 实现直驱系统的运动性能优 化。论文的结构如下, 第 1 节介绍数字孪生系统的 方案框架; 第 2 节针对直驱系统的线性环节和非线 性特性建立集总参数和非线性参数混合模型; 第 3 节对数字孪生模型的建模方法和模型同步策略进行 了介绍; 第 4 节着重分析了基于数字孪生模型开发 复合控制策略的设计过程, 并给出了详细计算步骤; 通过第 5 节的实验验证所提方法在惯量摄动下的有 效性和优越性; 第 6 节进行总结和展望。

\section{1 数字孪生系统开发框架}

基于数字孪生模型实现直驱系统的高精度控 制, 旨在完成从物理机器设备到数字孪生模型的数 据映射, 通过参数同步策略更新数字孪生模型特性, 从而优化控制策略, 实现对物理实体伺服控制参数 的循环迭代。据此提出如图 1 所示的数字孪生系统 开发框架, 框架由四个部分组成, 分别是直驱系统 物理实体、数字孪生模型、模型同步算法和复合控 制策略。 


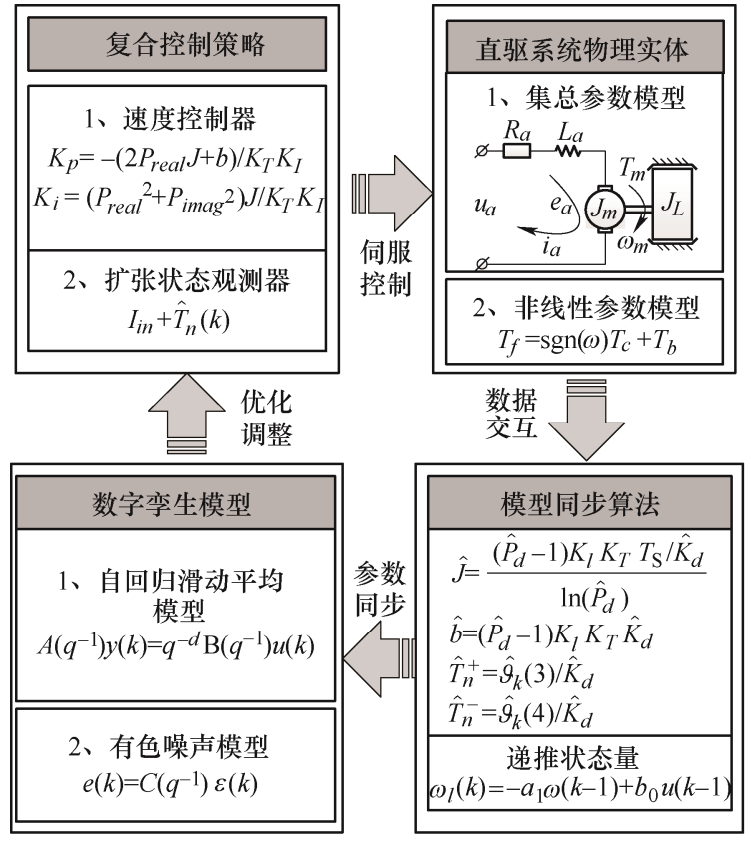

图 1 数字孪生系统开发框架

在物理模型建模阶段, 用集总参数模型描述线 性环节，用非线性参数模型描述非线性特征，从而 建立了基于动力学特性、非线性摩擦特性、伺服控 制回路以及信号采集环节的数字孪生模型。

在数字孪生模型构建阶段, 通过建立包含有色 噪声激励的自回归滑动平均模型实现对物理模型的 确定解析描述, 利用有色噪声模型描述直驱系统的 未建模动态特性, 提高模型的拟实度, 实现物理实 体参数与数字孪生模型参数的匹配。

在模型参数同步阶段, 通过采集物理实体传感 器和控制器输出数据, 基于递推增广最小二乘算法 实现数字孪生模型的参数同步以及数字孪生模型的 状态更新。

在复合控制策略循环优化和在线调整阶段, 为 了提高系统惯量摄动抑制能力, 提出了性能指标约 束的速度控制器和扩张状态卡尔曼滤波器, 利用数 字孪生模型数据对复合控制器进行优化调整, 从而 实现直驱系统在惯量摄动下的鲁棒性和运动控制 性能。

\section{2 直驱系统物理实体}

制造装备的种类千差万别, 如机械臂、数控车 床、精密指向平台和直线定位装置等, 其中力矩 电机直驱系统是制造装备广泛使用的基本运动 单元。

为了提高直驱部件的建模精度, 提出建立基于 集总参数模型和非线性参数模型相结合的混合模
型。建立传动轴系的统一模型表达范式需要充分结 合集总参数和非线性参数的优点, 用集总参数描述 物理结构的共性动力学特征, 用非线性参数描述个 性物理参数。

集总参数模型可以对制造装备物理结构耦合模 型进行线性化描述, 包含电气、驱动和传感三个环 节，能代表大部分制造装备的动力学特性。其中电 气部分需要考虑驱动器电流环带宽和反电动势的影 响等; 驱动部分需要考虑电动机和负载的惯量、回 转结合部产生的摩擦等; 传感部分主要考虑传感器 的测量精度和量化噪声。

非线性参数主要用于描述状态随着物理系统的 角位置、角速度等时变因素而变化的非线性因素, 例如数值与速度相关的摩擦力矩。

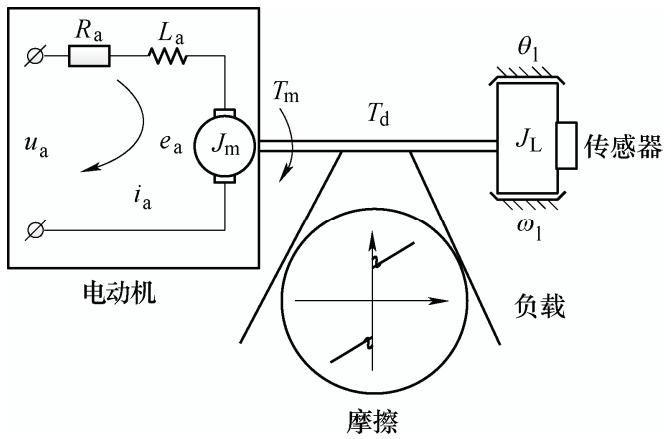

图 2 直驱部件模型示意图

图 2 为直驱部件模型示意图, 描述了电气环节、 驱动环节和传感装置的相互关系, 图中符号定义如 下: $L_{\mathrm{a}}$ 为电枢电感、 $R_{\mathrm{a}}$ 为电枢电阻、 $i_{\mathrm{a}}$ 为电枢电流、 $u$ 为电枢电压、 $e_{\mathrm{a}}$ 为反电动势、 $K_{\mathrm{T}}$ 为转矩系数、 $T_{\mathrm{d}}$ 为等效到电动机端的非线性干扰力矩、 $J_{\mathrm{m}}$ 为电动机 转子转动惯量、 $T_{\mathrm{m}}$ 为电动机输出转矩、 $\omega_{1}$ 为角速度、 $J_{1}$ 为负载转动惯量、 $\theta_{1}$ 为角位置。

下面利用集总参数模型和非线性参数模型共同 描述直驱部件的动力学特性。

\section{1 集总参数模型}

利用集总参数模型对直驱部件机电特性进行线 性化描述, 建立等效电动机模型和量化噪声模型, 为系统整体动力学分析和计算奠定基础。

得到系统线性动力学方程为

$$
\begin{gathered}
L_{\mathrm{a}} \frac{\mathrm{d} i_{\mathrm{a}}}{\mathrm{d} t}+R_{\mathrm{a}} i_{\mathrm{a}}=u_{\mathrm{a}}-e_{\mathrm{a}} \\
T_{m}=K_{T} i_{a} \\
\left(J_{\mathrm{m}}+J_{1}\right) \frac{\mathrm{d} \omega_{1}}{\mathrm{~d} t}=\left(T_{\mathrm{m}}-T_{\mathrm{d}}\right)
\end{gathered}
$$

受制于工艺水平和传感器制造精度, 传感器在 测量过程中不可避免地会引入量化噪声、随机误 
差、测量误差等因素, 对伺服控制的精度和稳定 性造成影响。量化噪声模型主要分为了 DA 数模转 换量化噪声、角位置测量量化噪声和角速率测量 量化噪声。

DA 模块的实际输出电压可以表示为

$$
I_{\text {in }}^{\prime}(k)=I_{\text {in }}(k)+\tilde{I}_{\text {in }}(k)
$$

式中, $I_{\text {in }}^{\prime}(k)$ 是实际输出电压, $I_{\text {in }}(k)$ 是电压指令, $\tilde{I}_{\mathrm{in}}(k)$ 为电压噪声, 噪声的分布和大小取决于数模转 换的精度。

假设输出电压的噪声分布近似为均匀的概率分 布, 可以获得数模转换噪声的方差为

$$
R_{\tilde{I}_{\text {in }}}=E\left\{\left(\tilde{I}_{\text {in }}-E\left[\tilde{I}_{\text {in }}\right]\right)^{2}\right\}=\left(\delta \tilde{I}_{\text {in }}\right)^{2} / 12
$$

式中, $\delta \tilde{I}_{\text {in }}$ 为 $\mathrm{DA}$ 转换精度。

同理, 根据传感器的分辨率可以得到角位置测 量量化噪声和角速率测量量化噪声的方差分别为

$$
R_{\tilde{\theta}_{1}}=\left(\delta \tilde{\theta}_{1}\right)^{2} / 12 \quad R_{\tilde{\omega}_{1}}=\left(\delta \tilde{\omega}_{1}\right)^{2} / 12
$$

式中, $\delta \tilde{\theta}_{1}$ 和 $\delta \tilde{\omega}_{1}$ 是角位置和角速度传感器的分 辨率。

\section{2 非线性参数模型}

非线性参数模型主要对集总参数模型的未建模 特征进行建模, 提高模型的拟实度。摩擦是运动部 件中最普遍存在的非线性因素, 主要包括了电动机 电刷摩擦和轴承摩擦, 是非线性参数模型的主要建 模对象。库伦和黏滞摩擦模型的摩擦曲线能基本体 现摩擦的主要特征, 又具有结构形式简单和参数辨 识容易等优点, 在实际工程中应用广泛。该摩擦模 型的表达式为

$$
\begin{gathered}
T_{\mathrm{f}}=\operatorname{sgn}(\omega) T_{\mathrm{c}}+T_{\mathrm{b}} \\
T_{\mathrm{b}}=b \omega_{1}
\end{gathered}
$$

式中, $T_{\mathrm{c}}$ 为库伦摩擦力, $T_{\mathrm{b}}$ 为黏滞摩擦力, $b$ 为黏 滞摩擦系数。

\section{3 数字孪生模型及模型同步算法}

为了实现对直驱系统物理实体的虚拟映射, 研 究数字孪生模型及其模型同步算法。利用自回归滑 动平均模型对直驱系统物理实体模型进行匹配, 通 过增加有色噪声模型作为未建模动态特性的描述, 提高模型辨识精度。进而基于递推增广最小二乘算 法, 通过采集直驱部件的状态数据, 实现模型参数 的更新和同步。主要分为数字孪生模型构建和模型 同步算法两个部分。

\section{1 数字孪生模型构建}

用确定解析模型来描述数字孪生模型, 通过对 集总参数和非线性参数中的线性部分的整合, 实现 模型参数与实际物理参数的对应，而利用增广模型 来描述未建模动态和非线性特征。

在实际系统中, 通过采集物理实体的输入力矩 和运动学反馈数据, 可以建立平稳时间序列来描述 运动部件等价系统的完整模型, 因此力矩电机直驱 系统的数字孪生模型可以含有有色噪声项的自回归 滑动平均模型来描述, 即

$$
\begin{gathered}
A\left(q^{-1}\right) y(k)=q^{-d} B\left(q^{-1}\right) u(k)+C\left(q^{-1}\right) \varepsilon(k) \\
A\left(q^{-1}\right)=1+a_{1} q^{-1}+a_{2} q^{-2}+\cdots+a_{n_{\mathrm{a}}} q^{-n_{\mathrm{a}}} \\
B\left(q^{-1}\right)=b_{0}+b_{1} q^{-1}+b_{2} q^{-2}+\cdots+b_{n_{\mathrm{b}}} q^{-n_{\mathrm{b}}} \\
C\left(q^{-1}\right)=1+c_{1} q^{-1}+c_{2} q^{-2}+\cdots+c_{n_{\mathrm{c}}} q^{-n_{\mathrm{c}}}
\end{gathered}
$$

增广模型用白噪声 $\varepsilon(k)$ 驱动的有色噪声模型 来描述，表达为

$$
e(k)=C\left(q^{-1}\right) \varepsilon(k)
$$

因此, 数字孪生模型具有以下形式

$$
\begin{aligned}
& y(k)=-a_{1} y(k-1)-\ldots-a_{n_{\mathrm{a}}} y\left(k-n_{\mathrm{a}}\right)+ \\
& b_{0} u(k-d)+\ldots+b_{n_{\mathrm{b}}} u\left(k-d-n_{\mathrm{b}}\right)+e(k)
\end{aligned}
$$

首先对摩擦力进行线性化处理, 将库伦摩擦力 矩等效到输入电压端, 则根据式(5)和(6)定义扰动力 矩表达式为

$$
T_{\mathrm{n}}^{+}=T_{\mathrm{c}}^{+} /\left(K_{\mathrm{I}} K_{\mathrm{T}}\right), \quad T_{\mathrm{n}}^{-}=T_{\mathrm{c}}^{-} /\left(K_{\mathrm{I}} K_{\mathrm{T}}\right)
$$

式中, $T_{\mathrm{c}}^{+}$和 $T_{\mathrm{c}}^{-}$代表正/负向库伦摩擦力, $K_{\mathrm{I}}$ 代表驱 动器的转换系数。

将摩擦力转化为速度的线性函数, 定义

$$
\begin{aligned}
& \left\{\begin{array}{l}
\text { 正向速度: } \varpi^{+}(\omega)=\frac{1}{2} \sigma(\omega)(1+\sigma(\omega)) \\
\text { 反向速度: } \varpi^{-}(\omega)=-\frac{1}{2} \sigma(\omega)(1-\sigma(\omega))
\end{array}\right. \\
& \sigma(\omega)= \begin{cases}1 & \omega>0 \\
-1 & \omega \leqslant 0\end{cases}
\end{aligned}
$$

综合式(10)和(11), 扰动力矩可以写为

$$
T_{\mathrm{n}}(\omega)=\varpi^{+}(\omega) T_{\mathrm{n}}^{+}+\varpi^{-}(\omega) T_{\mathrm{n}}^{-}
$$

构建以摩擦为主要非线性特征的动力学系统方 程, 结合式(1), 直驱系统的动力学方程可以表示为

$$
J \frac{\mathrm{d} \omega_{1}}{\mathrm{~d} t}=K_{\mathrm{T}} K_{\mathrm{I}}\left(I_{\text {in }}-T_{\mathrm{n}}\right)-b \omega_{1}
$$

式中, $J=J_{\mathrm{m}}+J_{1}$ 为等效惯量, $I_{\mathrm{in}}$ 为输入电压, 满 足 $i_{\mathrm{a}}=K_{\mathrm{I}} I_{\text {in }}$ 。 
实际物理系统的传递函数表达式为

$$
\omega_{1}(s)=\frac{K\left(I_{\text {in }}-T_{\mathrm{n}}\right)}{s+P}
$$

式中, $K=K_{\mathrm{T}} K_{\mathrm{I}} / J, P=b / J$ 。

式(14)的状态空间方程表达式为

$$
\begin{aligned}
{\left[\begin{array}{c}
\dot{\theta}_{1}(t) \\
\dot{\omega}_{1}(t)
\end{array}\right]=\boldsymbol{A}\left[\begin{array}{c}
\theta_{1}(t) \\
\omega_{1}(t)
\end{array}\right]+\left[\begin{array}{ll}
\boldsymbol{B} & -\boldsymbol{B}
\end{array}\right]\left[\begin{array}{c}
I_{\mathrm{in}} \\
T_{\mathrm{n}}
\end{array}\right] } \\
\boldsymbol{A}=\left[\begin{array}{cc}
0 & 1 \\
0 & -P
\end{array}\right] \quad \boldsymbol{B}=\left[\begin{array}{c}
0 \\
K
\end{array}\right]
\end{aligned}
$$

可以得到式(15)在零阶保持器条件下的离散传 递函数模型为

$$
\begin{gathered}
{\left[\begin{array}{c}
\theta_{1}(k+1) \\
\omega_{1}(k+1)
\end{array}\right]=\left[\begin{array}{cc}
1 & \left(1-e^{-P T_{\mathrm{s}}}\right) / P \\
0 & e^{-P T_{\mathrm{s}}}
\end{array}\right]\left[\begin{array}{l}
\theta_{1}(k) \\
\omega_{1}(k)
\end{array}\right]+} \\
{\left[\begin{array}{c}
K\left(T_{\mathrm{s}}+\left(e^{-P T_{\mathrm{s}}}-1\right) / P\right) / P \\
K\left(1-e^{-P T_{\mathrm{s}}}\right) / P
\end{array}\right] I_{\mathrm{in}}(k)}
\end{gathered}
$$

结合式(12)和(16), 得到包含有摩擦力矩的速度 预测差分方程为

$$
\begin{gathered}
\omega_{1}(k)=P_{\mathrm{d}} \omega_{1}(k-1)+K_{\mathrm{d}} I_{\text {in }}(k-1)-K_{\mathrm{d}} T_{\mathrm{n}}(k-1)= \\
{\left[\begin{array}{c}
\omega_{1}(k-1) \\
I_{\text {in }}(k-1) \\
-\varpi^{+}(\omega(k-1)) \\
-\varpi^{-}(\omega(k-1))
\end{array}\right]^{T}\left[\begin{array}{c}
P_{\mathrm{d}} \\
K_{\mathrm{d}} \\
K_{\mathrm{d}} T_{\mathrm{n}}^{+} \\
K_{\mathrm{d}} T_{\mathrm{n}}^{-}
\end{array}\right]}
\end{gathered}
$$

式中, $K_{\mathrm{d}}=K\left(1-e^{-P T_{\mathrm{s}}}\right) / P, P_{\mathrm{d}}=e^{-P T_{\mathrm{s}}}$ 。

图 3 为根据式 (17) 绘制的直驱部件数字孪生 模型原理框图, 通过离散化处理将直驱部件动力学 模型转化为自回归滑动平均模型的形式, 实现了对 直驱部件数字孪生模型的描述。

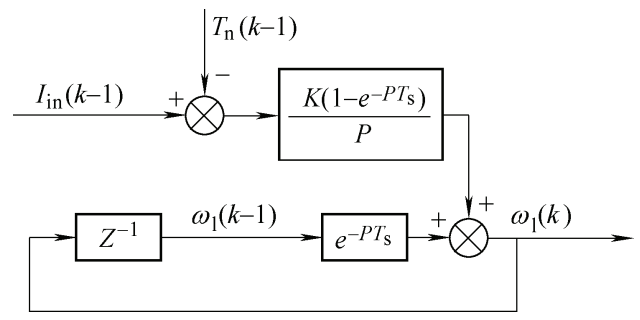

图 3 直驱部件数字孪生模型原理框图

\section{2 模型参数同步算法}

数字孪生模型的参数容易退化, 并受到物理实 体不同操作和环境条件的影响, 数字孪生的技术障 碍在于如何实现其动态模型更新以及与物理系统的 响应保持一致。为了使数字孪生模型成为物理实体 的精确镜像, 提出了一种基于递推增广最小二乘算 法的模型更新方案, 研究思路如图 4 所示。将模型 修正视为一个优化问题, 目标函数是使数字孪生模 型模拟的动态响应与物理系统测量的瞬时响应之间
的误差最小。

首先根据运动部件集总参数模型和非线性参数 模型提取出关键机械特性参数和状态参数, 将主要 参数作为系统辨识模型, 而用增广模型来描述系统 的未建模动态和噪声等因素, 提高辨识模型的精确 度; 然后根据辨识模型阶次和增广模型阶次构建辨 识对象的运动差分方程, 利用在线辨识方法实现对 集总参数和增广参数的估计, 实现制造装备运动状 态的更新。

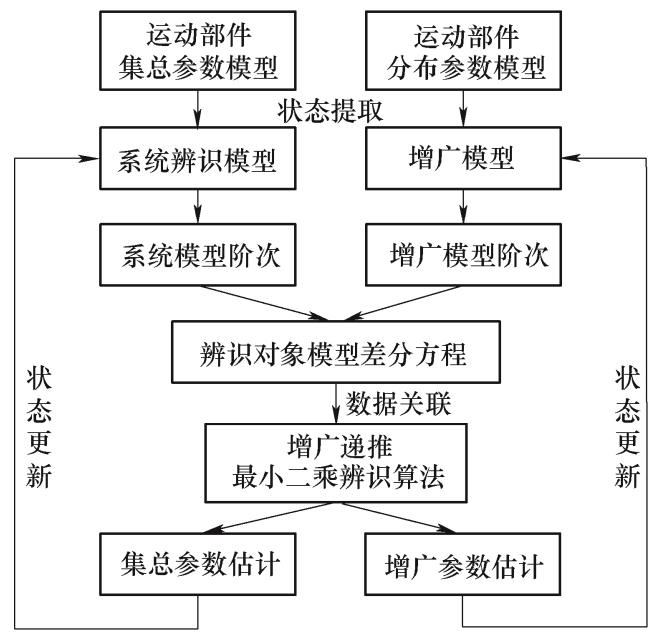

图 4 数字孪生模型参数同步策略示意图

提出一种基于确定解析模型的递推增广最小二 乘自适应参数辨识算法, 该方法具有收玫速度快、 辨识精度高和抗干扰能力强的特点。利用集总参数 模型和非线性参数模型构造参数辨识所需要的传递 函数描述形式, 对非线性摩擦力矩进行线性化处理, 基于线性化后的转速预测差分方程, 构造惯量和摩 擦特性参数的辨识方程。

为了实现数字孪生模型参数的迭代更新, 需要 构造递推状态量, 利用递推增广最小二乘自适应辨 识算法, 通过数据关联, 实现集总参数的自适应更 新。将式(17)变换为

$$
\left\{\begin{array}{l}
\omega_{1}(k)=-a_{1} \omega(k-1)+b_{0} \boldsymbol{u}(k-1) \\
a_{1}=-P_{\mathrm{d}} \boldsymbol{b}_{0}=\left[\begin{array}{lll}
K_{\mathrm{d}} & K_{\mathrm{d}} T_{\mathrm{n}}^{+} & K_{\mathrm{d}} T_{\mathrm{n}}^{-}
\end{array}\right] \\
\boldsymbol{u}(k-1)=\left[\begin{array}{lll}
I_{\mathrm{in}}(k-1) & -\varpi^{+}(\omega) & -\varpi^{-}(\omega)
\end{array}\right]^{\mathrm{T}}
\end{array}\right.
$$

结合式(17)和(18), 可以得到运动控制部件模型 参数的增广最小二乘递推计算公式为

$$
\left\{\begin{array}{l}
\hat{\lambda}(k+1)=\hat{\lambda}(k)+K_{\mathrm{rls}}(k+1)\left[\omega_{1}(k+1)-\boldsymbol{\varphi}^{\mathrm{T}}(k+1) \hat{\lambda}(k)\right] \\
\boldsymbol{K}_{\mathrm{rls}}(k+1)=\frac{\boldsymbol{P}_{\mathrm{rls}}(k) \boldsymbol{\varphi}(k+1)}{1+\boldsymbol{\varphi}^{\mathrm{T}}(k+1) \boldsymbol{P}_{\mathrm{rls}}(k) \boldsymbol{\varphi}(k+1)} \\
\boldsymbol{P}_{\mathrm{rls}}(k+1)=\left[\boldsymbol{I}-\boldsymbol{K}_{\mathrm{rls}}(k+1) \boldsymbol{\varphi}^{\mathrm{T}}(k+1)\right] \boldsymbol{P}_{\mathrm{rls}}(k)
\end{array}\right.
$$


式中, $\boldsymbol{\varphi}^{\mathrm{T}}(k)=\left[-\omega_{1}(k-1), u(k-1)\right] 、 \hat{\lambda}^{\mathrm{T}}=\left[a_{1}, b_{0}\right]$ 、 $\boldsymbol{K}_{\mathrm{rls}}(k+1)$ 和 $\boldsymbol{P}_{\mathrm{rls}}(k+1)$ 分别代表回归矩阵、参数向 量、递推增益和估计偏差的协方差矩阵。

根据式(17), 得到参数估计方程为

$$
\left\{\begin{array}{l}
\hat{P}_{\mathrm{d}}=\hat{\lambda}_{\mathrm{k}}(1) \\
\hat{K}_{\mathrm{d}}=\hat{\lambda}_{\mathrm{k}}(2) \\
\hat{T}_{\mathrm{n}}^{+}=\hat{\lambda}_{\mathrm{k}}(3) / \hat{K}_{\mathrm{d}} \\
\hat{T}_{\mathrm{n}}^{-}=\hat{\lambda}_{\mathrm{k}}(4) / \hat{K}_{\mathrm{d}} \\
\hat{J}=\left(\hat{P}_{\mathrm{d}}-1\right) K_{\mathrm{I}} K_{\mathrm{T}} T_{\mathrm{s}} / \hat{K}_{\mathrm{d}} / \ln \left(\hat{P}_{\mathrm{d}}\right) \\
\hat{b}=\left(\hat{P}_{\mathrm{d}}-1\right) K_{\mathrm{I}} K_{\mathrm{T}} / \hat{K}_{\mathrm{d}}
\end{array}\right.
$$

式中, $\hat{P}_{\mathrm{d}} 、 \hat{K}_{\mathrm{d}} 、 \hat{T}_{\mathrm{n}}^{+} 、 \hat{T}_{\mathrm{n}}^{-} 、 \hat{J}$ 和 $\hat{b}$ 分别的代表了 $P_{\mathrm{d}}$ 、 $K_{\mathrm{d}} 、 T_{\mathrm{n}}^{+} 、 T_{\mathrm{n}}^{-} 、 J$ 和 $b$ 的估计值, 为了加快收玫速 度, 一般预先计算参数的迭代初始值。

\section{4 复合控制策略}

为了实现数字孪生驱动的伺服控制系统设计, 提出如图 5 所示的伺服控制实施途径, 包含实际物 理系统、虚拟物理系统和参数同步三个环节。虚拟物 理系统为集总参数模型和非线性参数模型结合的混合 模型, 通过与实际物理系统的数据交互实现模型参数 的计算和更新, 从而辅助实际物理系统的伺服设计过 程, 优化控制参数, 提高物理实体的运动性能。

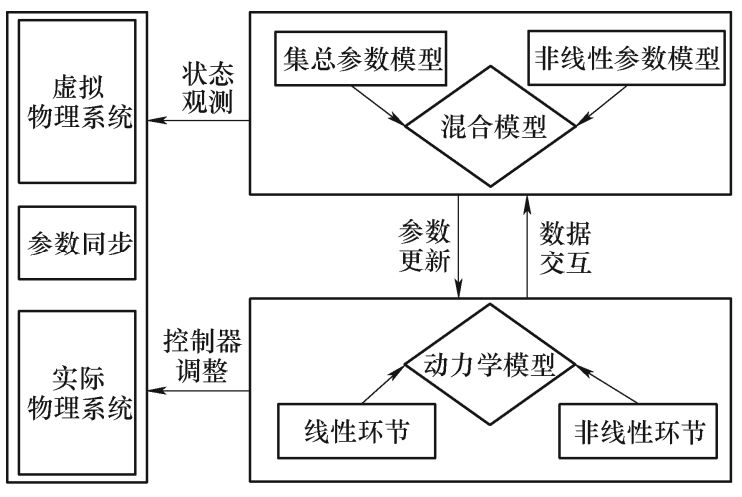

图 5 数字孪生驱动的伺服控制实施途径

根据图 5 所示的伺服控制实施途径, 设计了 图 6 所示的数字孪生驱动复合控制原理图, 描述了 实际物理系统、数字孪生模型和复合控制策略的 联系。

复合控制策略主要由速度控制器和扩张状态卡 尔曼滤波器两个部分组成, 速度控制器用于保证速 度回路的稳定性, 并对实际物理系统的性能进行线 性设计。扩张状态卡尔曼滤波器则用于估计非线性 扰动力矩, 将估计值通过前馈的方式补偿到控制器
输出端, 提高系统扰动抑制能力。

通过数据交互和模型同步算法, 实现数字孪生 模型与实际物理系统关键参数的实时同步, 辅助复 合控制策略的在线优化和调整。

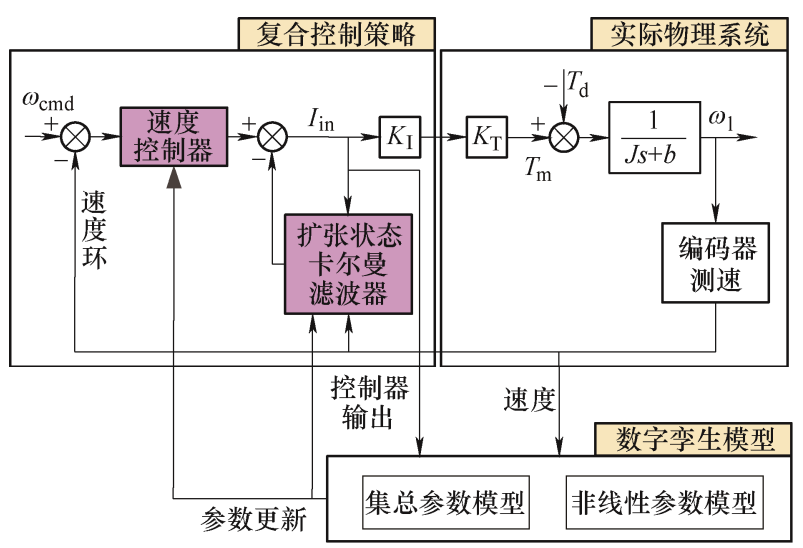

图 6 数字孪生驱动的复合控制原理图

\section{1 性能指标约束的速度控制器设计}

根据数字孪生模型参数同步的结果, 获取实际 物理系统的状态参数, 开展基于性能指标约束的速 度控制器设计, 提高直驱系统在惯量摄动情况下的 伺服性能。

对式(13)所示的动力学系统进行速度环比例积 分控制器的设计, 首先定义

$$
\omega_{\mathrm{s}}=\int_{0}^{t}\left(\omega_{\mathrm{cmd}}-\omega_{1}\right) \mathrm{d} \tau
$$

式中, $\omega_{\mathrm{cmd}}$ 是速度环指令。

比例积分控制器的输出为

$$
I_{\text {in }}=K_{\mathrm{p}}\left(\omega_{\mathrm{cmd}}-\omega_{1}\right)+K_{\mathrm{i}} \omega_{\mathrm{s}}
$$

式中, $K_{\mathrm{p}}$ 为比例系数, $K_{\mathrm{i}}$ 为积分系数。

结合式(13)、(21)和(22), 可以建立速度环微分 方程组为

$\left\{\begin{array}{l}\dot{\omega}_{1}=-\left(K_{\mathrm{T}} K_{\mathrm{I}} K_{\mathrm{p}}+b\right) \omega_{1} / J+K_{\mathrm{T}} K_{\mathrm{I}} K_{\mathrm{p}} \omega_{\mathrm{cmd}} / J+ \\ K_{\mathrm{T}} K_{\mathrm{I}} K_{\mathrm{i}} \omega_{\mathrm{s}} / J-K_{\mathrm{T}} K_{\mathrm{I}} T_{\mathrm{n}} / J \\ \dot{\omega}_{\mathrm{s}}=-\omega_{1}+\omega_{\text {cmd }}\end{array}\right.$

得到速度闭环系统状态方程为

$$
\begin{gathered}
{\left[\begin{array}{c}
\dot{\omega}_{1} \\
\dot{\omega}_{\mathrm{s}}
\end{array}\right]=\left[\begin{array}{cc}
-\left(K_{\mathrm{T}} K_{\mathrm{I}} K_{\mathrm{p}}+b\right) / J & N K_{\mathrm{T}} K_{\mathrm{I}} K_{\mathrm{i}} / J \\
-1 & 0
\end{array}\right]\left[\begin{array}{l}
\omega_{1} \\
\omega_{\mathrm{s}}
\end{array}\right]+} \\
{\left[\begin{array}{cc}
N K_{\mathrm{T}} K_{\mathrm{I}} K_{\mathrm{p}} / J & 1 / J \\
1 & 0
\end{array}\right]\left[\begin{array}{c}
\omega_{\mathrm{cmd}} \\
T_{\mathrm{n}}
\end{array}\right]}
\end{gathered}
$$

闭环系统矩阵为

$$
\boldsymbol{A}_{\mathrm{c}}=\left[\begin{array}{cc}
-\left(K_{\mathrm{T}} K_{\mathrm{I}} K_{\mathrm{p}}+b\right) / J & N K_{\mathrm{T}} K_{\mathrm{I}} K_{\mathrm{i}} / J \\
-1 & 0
\end{array}\right]
$$

提出基于系统调整时间和阻尼的控制器设计方 
法。系统的动态响应取决于状态空间方程的零极点 位置, 确定系统调整时间 $t_{\mathrm{s}}$ 和阻尼 $\xi$ 后, 可以根据 闭环系统矩阵的极点分布, 求解比例系数和积分系 数。假设两个共轭根为 $\lambda_{1}$ 和 $\lambda_{2}$, 则极点位置为

$$
\lambda_{1,2}=P_{\text {real }} \pm P_{\text {imag }} j
$$

式中, $P_{\text {real }}=-\xi \omega_{\mathrm{n}}, P_{\text {imag }}=\omega_{\mathrm{n}} \sqrt{1-\xi^{2}}$ 。

可知, 闭环系统的两个共轭根满足下式

$$
\left|\begin{array}{cc}
\lambda+\left(K_{\mathrm{T}} K_{\mathrm{l}} K_{\mathrm{p}}+b\right) / J & -N K_{\mathrm{T}} K_{\mathrm{I}} K_{\mathrm{i}} / J \\
1 & \lambda
\end{array}\right|=0
$$

结合式(26)和(27), 可以求得控制器参数为

$$
K_{\mathrm{p}}=\frac{-2 P_{\text {real }} J-b}{K_{\mathrm{T}} K_{\mathrm{I}}} \quad K_{\mathrm{i}}=\frac{\left(P_{\text {real }}^{2}+P_{\text {imag }}^{2}\right) J}{K_{\mathrm{T}} K_{\mathrm{I}}}
$$

通过数字孪生模型的参数同步, 动态配置 $K_{\mathrm{p}}$ 和 $K_{\mathrm{i}}$ 实现对闭环零极点的任意配置, 从而设计满足系 统响应时间和阻尼等性能的控制器参数。

\section{2 扩张状态卡尔曼滤波器设计}

直驱部件的未建模特性和非线性摩擦是影响伺 服性能的主要因素, 为了提高直驱部件的扰动抑制 能力, 基于数字孪生模型提出一种扩张状态卡尔曼 滤波器, 根据数字孪生模型参数变化, 适当调整观 测器性能，抑制非线性因素对运动性能的影响。

对式(15)所示的直驱部件动力学系统进行离散 化处理, 可以得到离散状态空间方程为

$$
\left[\begin{array}{c}
\theta_{1}(k+1) \\
\omega_{1}(k+1)
\end{array}\right]=\boldsymbol{A}_{\mathrm{d}}\left[\begin{array}{c}
\theta_{1}(k+1) \\
\omega_{1}(k+1)
\end{array}\right]+\left[\begin{array}{ll}
\boldsymbol{B}_{\mathrm{d}} & -\boldsymbol{B}_{\mathrm{d}}
\end{array}\right]\left[\begin{array}{c}
I_{\text {in }} \\
T_{\mathrm{n}}
\end{array}\right]
$$

式中, $\boldsymbol{A}_{\mathrm{d}}=e^{A T_{\mathrm{s}}}, \boldsymbol{B}_{\mathrm{d}}=\int_{0}^{T_{\mathrm{s}}} e^{A \tau} \mathrm{d} \tau \cdot \boldsymbol{B}$ 。

根据式(29)建立包含动力学模型和噪声模型的 离散扩展状态空间方程，得到

$$
\left\{\begin{array}{l}
{\left[\begin{array}{c}
\theta_{1}(k+1) \\
\omega_{1}(k+1) \\
T_{\mathrm{n}}(k+1)
\end{array}\right]=\boldsymbol{A}_{\mathrm{dk}}\left[\begin{array}{c}
\theta_{1}(k) \\
\omega_{1}(k) \\
T_{\mathrm{n}}(k)
\end{array}\right]+\boldsymbol{B}_{\mathrm{dk}}\left[I_{\mathrm{in}}(k)\right]+\boldsymbol{W}_{\mathrm{dk}}\left[\begin{array}{c}
\tilde{I}_{\mathrm{in}}(k) \\
\tilde{T}_{\mathrm{n}}(k)
\end{array}\right]} \\
{\left[\begin{array}{c}
\theta_{1}^{\prime}(k) \\
\omega_{1}^{\prime}(k)
\end{array}\right]=\boldsymbol{C}_{\mathrm{dk}}\left[\begin{array}{c}
\theta_{1}(k) \\
\omega_{1}(k) \\
T_{\mathrm{n}}(k)
\end{array}\right]+\boldsymbol{V}_{\mathrm{dk}}\left[\begin{array}{c}
\tilde{\theta}_{1}(k) \\
\tilde{\omega}_{1}(k)
\end{array}\right]}
\end{array}\right.
$$

式中, $\tilde{T}_{\mathrm{n}}$ 代表观测噪声

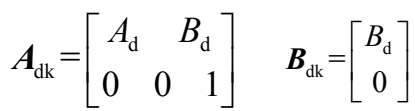

$$
\begin{aligned}
& \boldsymbol{C}_{\mathrm{dk}}=\left[\begin{array}{lll}
1 & 0 & 0 \\
0 & 1 & 0
\end{array}\right] \quad \boldsymbol{W}_{\mathrm{dk}}=\left[\begin{array}{cc}
B_{\mathrm{d}} & 0 \\
& 0 \\
0 & 1
\end{array}\right] \quad \boldsymbol{V}_{\mathrm{dk}}=\left[\begin{array}{ll}
1 & 0 \\
0 & 1
\end{array}\right]
\end{aligned}
$$

进一步, 得到扩张状态卡尔曼滤波器的形式为

$$
\begin{gathered}
{\left[\begin{array}{c}
\hat{\theta}_{1}(k) \\
\hat{\omega}_{1}(k) \\
\hat{T}_{\mathrm{n}}(k)
\end{array}\right]=\left(\boldsymbol{I}-\boldsymbol{L} \boldsymbol{C}_{\mathrm{dk}}\right) \boldsymbol{A}_{\mathrm{dk}}\left[\begin{array}{c}
\hat{\theta}_{1}(k-1) \\
\hat{\omega}_{1}(k-1) \\
\hat{T}_{\mathrm{n}}(k-1)
\end{array}\right]+\boldsymbol{L}\left[\begin{array}{c}
\theta_{1}^{\prime}(k) \\
\omega_{1}^{\prime}(k)
\end{array}\right]+} \\
\left(\boldsymbol{I}-\boldsymbol{L} \boldsymbol{C}_{\mathrm{dk}}\right) \boldsymbol{B}_{\mathrm{dk}}\left[I_{\mathrm{in}}(k-1)\right]
\end{gathered}
$$

增益 $\boldsymbol{L}$ 可以通过下列方程离线迭代求取

$$
\left\{\begin{array}{l}
\boldsymbol{P}(k \mid k-1)=\boldsymbol{A}_{\mathrm{dk}} \boldsymbol{P}(k \mid k-1) \boldsymbol{A}_{\mathrm{dk}}^{\mathrm{T}}+\boldsymbol{W R}_{\mathrm{w}} \boldsymbol{W}^{\mathrm{T}} \\
\boldsymbol{L}(k)=\boldsymbol{P}(k \mid k-1) \boldsymbol{C}_{\mathrm{dk}}^{\mathrm{T}}\left[\boldsymbol{R}_{\mathrm{v}}+\boldsymbol{C P}(k \mid k-1) \boldsymbol{C}_{\mathrm{dk}}^{\mathrm{T}}\right]^{-1} \\
\boldsymbol{P}(k \mid k 1)=\left[\boldsymbol{I}-\boldsymbol{L}(k) \boldsymbol{C}_{\mathrm{dk}}\right] \boldsymbol{P}(k \mid k-1)
\end{array}\right.
$$

式中, $\boldsymbol{P}$ 为协方差矩阵, $R_{\tilde{T}_{\mathrm{n}}}$ 为扰动力矩观测噪声方 差, $\boldsymbol{R}_{\mathrm{w}}=\operatorname{diag}\left\{R_{\tilde{I}_{\mathrm{in}}}, R_{\tilde{T}_{\mathrm{n}}}\right\} ， \boldsymbol{R}_{\mathrm{w}}=\operatorname{diag}\left\{R_{\tilde{\theta}_{1}}, R_{\tilde{\omega}_{1}}\right\}$ 。

通过扩张状态卡尔曼滤波器, 实现对位置、速 度和非线性扰动信号的估计, 采用前馈的方式补偿 到速度控制器输出端。因此, 控制器总输出为

$$
I_{\text {total }}=I_{\text {in }}+\hat{T}_{\mathrm{n}}(k)
$$

\section{3 复合控制算法设计步骤}

为了有助于伺服控制算法的工程实现, 提供复 合控制策略的详细设计和计算步骤, 提高设计效率。 步骤如下。

(1) 根据力矩电机及其负载的机械参数, 获取 式(1) (6)中的参数。

(2) 根据直驱系统性能要求, 确定速度闭环的 调整时间为 $t_{\mathrm{s}}$ 和闭环系统阻尼 $\xi$ 。

(3) 将直驱系统的参数代入式(26)和(28)中, 计 算得到比例积分控制器参数 $K_{\mathrm{p}}$ 和 $K_{\mathrm{i}}$ 。

(4) 将直驱系统的参数代入式(30)中, 得到包含 动力学模型和噪声模型的离散扩展状态空间方程矩 阵 $\boldsymbol{A}_{\mathrm{dk}}$ 和 $\boldsymbol{B}_{\mathrm{dk}}$ 。

(5) 根据传感器配置情况, 获取量测矩阵 $\boldsymbol{C}_{\mathrm{dk}}$ 。

(6) 预先选取扰动力矩观测噪声方差的值 $R_{\tilde{r}_{\mathrm{n}}}$, 经过式(32)所示的离线迭代方法, 计算得到扩张状 态卡尔曼滤波器的增益 $\boldsymbol{L}$ 。

(7) 如果系统出现振荡失稳的现象, 则返回步 骤(2), 增大调整时间。如果扰动抑制效果不佳, 则 返回到步骤(6), 按照由小至大的顺序, 重新选择 $R_{\tilde{T}_{\mathrm{n}}}$ 的值, 并重新计算 $\boldsymbol{L}$, 直到满足性能指标。

\section{5 试验验证}

为了验证基于数字孪生模型的复合控制策略的 有效性, 搭建力矩电机直驱系统实验测试系统, 利 用 $\mathrm{dSAPCE}$ 半实物仿真平台, 开展伺服性能测试实 
验, 主要定量测试以下三个方面的内容: 惯量摄动 下的直驱系统的数字孪生模型同步精度、速度正弦 指令跟随精度和速度阶跃指令响应特性。

\section{1 试验系统的建立}

搭建了如图 7 所示的力矩电动机直驱系统, 主要 由稀土永磁直流力矩电动机(型号：130LCX-2)、传动 轴系、可调节惯量盘、质量块、光电编码器(型号: Heidenhain RON285)、直流伺服驱动器 (型号: AMC30A8)、电源模块以及 dSPACE1103 半实物仿真 平台组成, 其中惯量盘上设计有可拆卸的质量块, 通 过调节质量块的个数和位置, 可以模拟负载惯量变化 的情况。该试验系统具备负载惯量调节、力矩电动机 驱控和控制器在线调试的功能, 满足对比试验的条件。

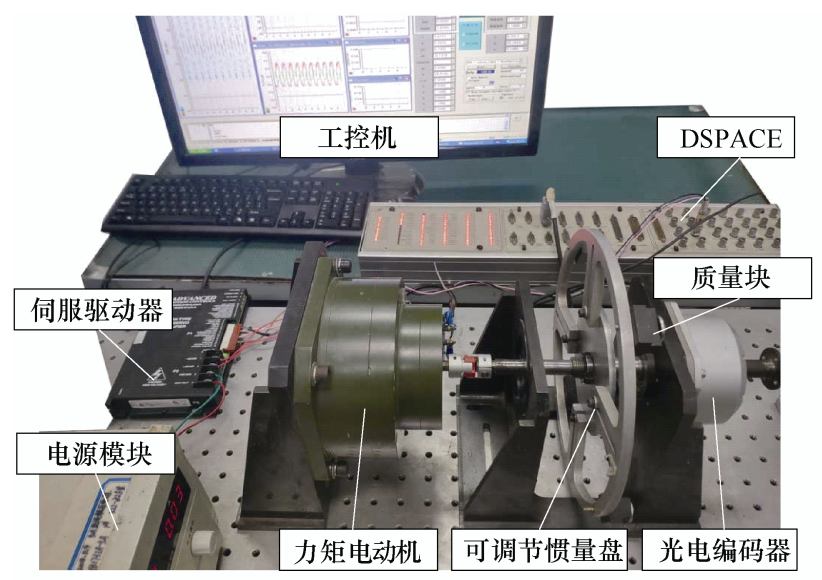

图 7 力矩电动机直驱系统

通过查阅试验设备的具体型号和关键参数, 整 理得到表 1 所示的伺服设计参数数值。

\section{表 1 伺服设计参数数值}

\begin{tabular}{cc}
\hline 设计参数 & 数值 \\
\hline 惯量 $J /\left(\mathrm{kg} \cdot \mathrm{m}^{2}\right)$ & (空载) $6.5 \times 10^{-3}$ \\
(带载) $8.9 \times 10^{-3}$ \\
黏滞摩擦系数 $b /\left(\mathrm{N} \cdot \mathrm{m} \cdot \mathrm{s} \cdot \mathrm{rad}^{-1}\right)$ & 0.256 \\
力矩系数 $K_{\mathrm{T}} /\left(\mathrm{N} \cdot \mathrm{m} \cdot \mathrm{A}^{-1}\right)$ & 0.73 \\
驱动器转换系数 $K_{\mathrm{I}} /(\mathrm{A} / \mathrm{V})$ & 1.13 \\
$\mathrm{DA}$ 量化噪声方差 $R_{\tilde{I}_{\mathrm{in}}} /\left(\mathrm{V}^{2}\right)$ & $2.0 \times 10^{-6}$ \\
位置测量噪声方差 $R_{\tilde{\theta}_{1}} /\left[\left({ }^{\circ}\right)^{2}\right]$ & $5.1 \times 10^{-10}$ \\
速度测量噪声方差 $R_{\tilde{\omega}_{1}} /\left[\left({ }^{\circ}\right)^{2} \cdot \mathrm{s}^{-2}\right]$ & $5.1 \times 10^{-4}$ \\
\hline
\end{tabular}

为了验证数字孪生驱动下复合控制策略(Digital twin driven PI controller with state augmented Kalman filter, DTPISAKF)的有效性, 另外设置了两组对照组控 制器, 分别是传统比例增益控制器(Constant gain PI controller, CGPI)和数字孪生驱动的比例增益控制器 (Digital twin driven PI controller, DTPI)。

试验过程描述为: 通过在实验中改变质量块的
数量和位置, 实现惯量盘惯量的增减, 验证在惯量 变化情况下所提数字孪生模型参数同步方法有效性 以及数字孪生驱动复合控制方法的优越性。首先在 空载条件下, 根据初始控制器参数实现对试验装置 的伺服控制, 运用 CGPI、DTPI 和 DTPISAKF 三种 控制器进行三组试验, 分别记录不同控制器作用下 的正弦指令跟随性能、阶跃响应性能以及数字孪生 模型参数同步的过程。之后调节惯量盘的质量块, 增大负载惯量, 重复上述三组试验。速度参考指令 的幅值为 $20 \%$, 频率为 $1 \mathrm{~Hz}$ 。

\section{2 复合控制器参数的设置}

为了增强伺服控制过程的平稳性, 需要离线获 取控制器参数的初始值, 根据表 1 的伺服设计参数 数值, 对控制器参数进行详细设计。图 5 中数字孪 生驱动的伺服控制原理图已经给出了复合控制器的 组成, 首先进行基于性能指标约束下的速度环控制 器设计。

(1) 获取直驱系统参数, 结果如表 1 所示。

(2) 根据前期调试经验, 设定直驱系统速度闭 环的调整时间为 $t_{\mathrm{s}}=0.1 \mathrm{~s}$, 闭环系统阻尼为 $\xi=0.707$, 则闭环系统的期望极点位置为

$$
\lambda_{1,2}=-30 \pm 30 j
$$

(3) 将参数代入式(26)和(28)中, 计算得到

$$
K_{\mathrm{p}}=0.207, K_{\mathrm{i}}=18.12
$$

(4) 将参数代入式(30)中, 得到包含动力学模型 和噪声模型的离散扩展状态空间方程矩阵为

$$
\begin{gathered}
A_{\mathrm{dk}}=\left[\begin{array}{ccc}
1 & 3.9 \times 10^{-2} & 6.3 \times 10^{-5} \\
0 & 0.96 & 0.12 \\
0 & 0 & 1
\end{array}\right] \\
\boldsymbol{B}_{\mathrm{dk}}=\left[\begin{array}{c}
6.3 \times 10^{-5} \\
0.12 \\
0
\end{array}\right]
\end{gathered}
$$

(5) 在试验中将光电编码器的测速信号作为速 度反馈信号, 则量测矩阵可以写为

$$
\boldsymbol{C}_{\mathrm{dk}}=\left[\begin{array}{lll}
0 & 1 & 0
\end{array}\right]
$$

(6) 在调试中选取扰动力矩观测噪声方差的值 为 $R_{\tilde{T}_{\mathrm{n}}}=0.01$, 经过式(32)所示的离线迭代方法, 计算 得到扩张状态卡尔曼滤波器的增益为

$$
\boldsymbol{L}=\left[\begin{array}{lll}
0.014 & 0.65 & 2.63
\end{array}\right]^{\mathrm{T}}
$$

将以上控制器参数作为基于数字孪生模型的高 精度伺服控制器的初始值, 开展传统算法与所提算 法的性能对比实验。 


\section{3 试验结果}

分析和对比惯量摄动下直驱系统的数字孪生模 型同步精度、速度正弦指令跟随精度和速度阶跃指 令响应特性。

\subsection{1 数字孪生模型同步精度}

通过与实际物理系统的速度信号和控制器输出 信号的交互, 同步算法实现对数字孪生模型参数的 辨识和更新。

图 8 和图 9 为空载和带载情况下数字孪生模型 参数收玫过程, 主要参数为等效转动惯量 $J$ 、黏滞 阻尼系数 $b$ 、以及正、负方向库伦摩擦 $T_{\mathrm{c}}^{+}$和 $T_{\mathrm{c}}^{-}$, 展现了模型参数由初始值收敛于真值的过程。
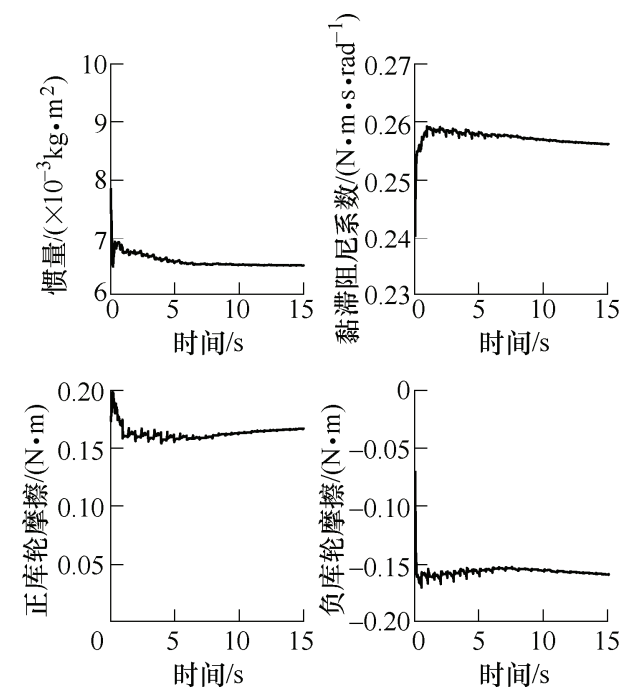

图 8 空载情况下数字孪生模型参数收敛过程
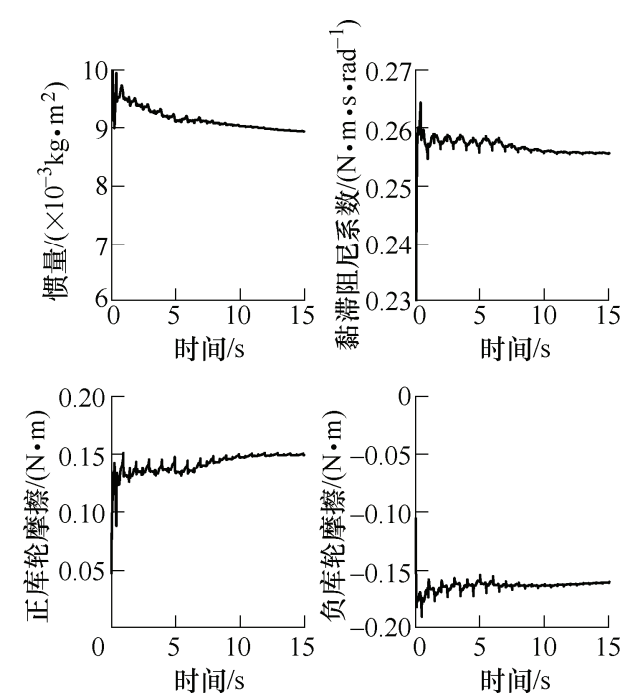

图 9 带载情况下数字孪生模型参数收敛过程

将数字孪生模型参数同步结果记录在表 2 中, 可以看出, 在负载摄动的情况下, 模型同步算法准 确检测到了负载惯量的增加, 等效转动惯量 $J$ 由 $6.5 \times 10^{-3} \mathrm{~kg} \cdot \mathrm{m}^{2}$ 增加到了 $8.9 \times 10^{-3} \mathrm{~kg} \cdot \mathrm{m}^{2}, b 、 T_{\mathrm{c}}^{+}$和
$T_{\mathrm{c}}^{-}$的数值变化不大, 说明了系统的摩擦特性没有发 生明显改变。以上数据分别从正面和侧面验证了数 字孪生模型参数同步方法的收敛性和有效性。

\section{表 2 数字孪生模型参数同步结果}

\begin{tabular}{ccc}
\hline 参数辨识结果 & 空载情况 & 带载情况 \\
\hline 惯量 $J /\left(\mathrm{kg} \cdot \mathrm{m}^{2}\right)$ & $6.5 \times 10^{-3}$ & $8.9 \times 10^{-3}$ \\
黏滞阻尼系数 $b /\left(\mathrm{N} \cdot \mathrm{m} \cdot \mathrm{s} \cdot \mathrm{rad}^{-1}\right)$ & 0.256 & 0.255 \\
正库伦摩擦 $T_{\mathrm{c}}^{+} /(\mathrm{N} \cdot \mathrm{m})$ & 0.0170 & 0.168 \\
负库伦摩擦 $T_{\mathrm{c}}^{-} /(\mathrm{N} \cdot \mathrm{m})$ & -0.163 & -0.165 \\
\hline
\end{tabular}

\subsection{2 速度正弦指令跟随精度}

直驱系统在惯量摄动情况下跟随速度正弦指 令，对比三种控制方法抵抗惯量摄动的能力。

空载情况下, DTPISAKF 的死区长度最短, 速 度残差幅值最低, 能有效克服非线性因素影响, 跟 随效果最好。图 10 和图 11 分别为空载时系统跟随 正弦指令的速度曲线及其残差曲线, 其中图例 “Design” 代表了速度闭环理论响应曲线。

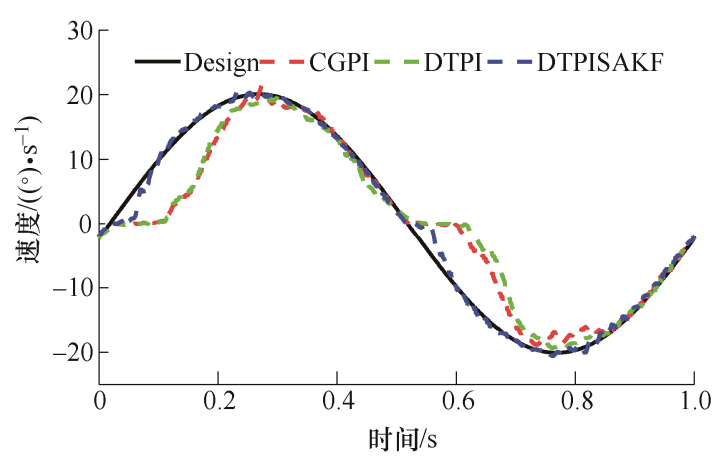

图 10 空载时系统跟随正弦指令的速度曲线

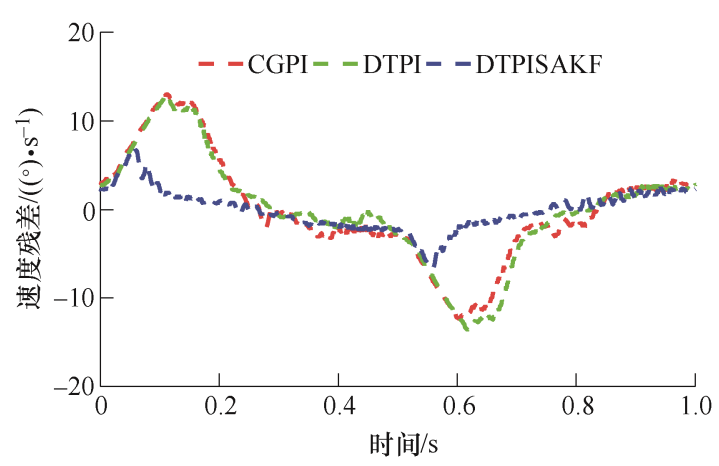

图 11 空载时系统跟随正弦指令的速度残差曲线

带载情况下, DTPISAKF 控制器性能最优, 依 旧能克服惯量变化的影响, 保持系统的动态性能, 而 CGPI 控制器表现出性能劣化现象。图 12 和图 13 分别为带载时系统跟随正弦指令的速度曲线及其残 差曲线。 


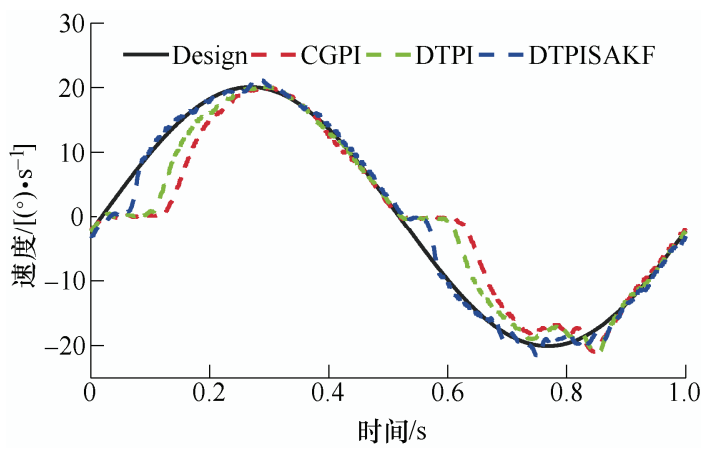

图 12 带载时系统跟随正弦指令的速度曲线

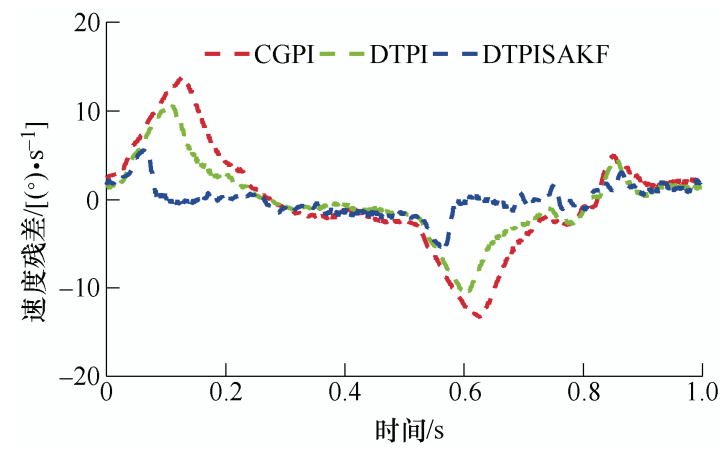

图 13 带载时系统跟随正弦指令的速度残差曲线

为了量化分析三种控制方法下的正弦响应特 性, 表 3 给出了系统速度跟随残差的均方根误差 (Root mean squared error, RMSE)统计表。可知, DTPISAKF 在惯量摄动下的跟随精度明显优于其他 控制器, 相对于传统控制器, RMSE 由 $5.88 \%$ 降低 为 $1.76 \%$, 衰减幅度达到了 $70 \%$ 。

表 3 系统速度跟随残差的均方根误差 $\left({ }^{\circ} / \mathrm{s}\right)$

\begin{tabular}{ccc}
\hline 控制方法 & 空载情况 & 带载情况 \\
\hline CGPI & 6.01 & 5.88 \\
DTPI & 6.06 & 4.12 \\
DTPISAKF & 2.30 & 1.76 \\
\hline
\end{tabular}

根据以上分析可以得到以下结论: 在定常系统 中, CGPI 和 DTPI 具有相近的控制效果, DTPISAKF 能有效补偿系统受到的非线性摩擦力矩, 提高速度 控制精度, 增强系统的非线性抑制能力; 在惯量变 化的时变系统中, CGPI 和 DTPI 控制器无法有效保 证系统的设计性能指标, 本文提出的 DTPISAKF 控 制器能够有效保证系统伺服控制精度, 提高系统鲁 棒性和扰动抑制能力。

\subsection{3 速度阶跃指令响应特性}

记录直流伺服系统在惯量摄动情况下的阶跃响 应曲线, 通过与设计响应曲线的对比, 评价三种控 制器作用下系统的伺服性能。

惯量摄动条件下, DTPISAKF 的阶跃响应曲线
均能够较好地拟合设计阶跃响应曲线, 系统调整时 间符合 $0.1 \mathrm{~s}$ 的指标要求。而传统控制方法的调整时 间为 $0.3 \mathrm{~s}$ 左右, 不满足设计指标。图 14 和图 15 分 别为空载和带载时系统的阶跃响应曲线。

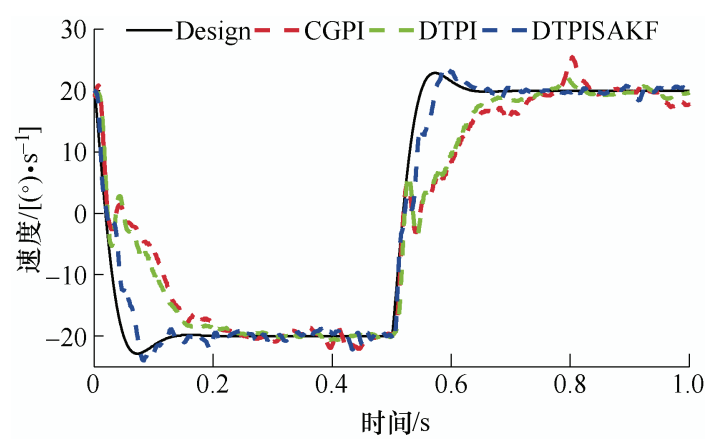

图 14 空载时系统阶跃响应曲线

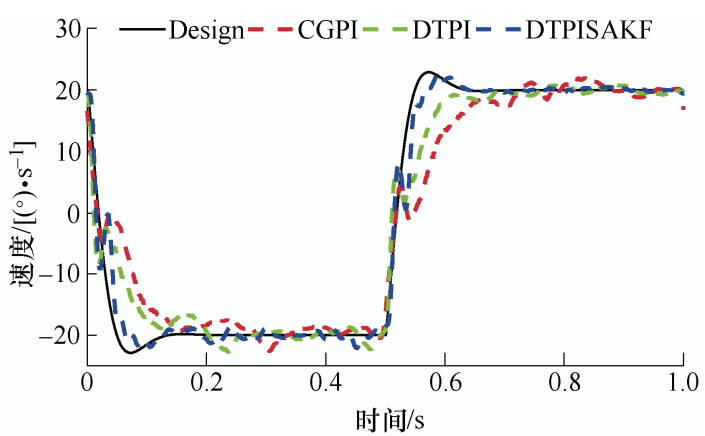

图 15 带载时系统阶跃响应曲线

为了定量分析系统在三种控制器作用下的阶跃 响应性能, 引入 Pearson 相关系数用于评价实际响 应曲线与理论响应曲线的拟合程度。Pearson 相关系 数是两个变量的协方差与标准差之积的比值, 用来 衡量两个变量之间的相关程度, 取值范围为 -1 到 1 , 数据拟合度越高, 相关系数越则趋近于 1 , 其计算 公式为

$$
r=\frac{\sum X Y-\left(\sum X \sum Y\right) / N}{\sqrt{\left(\sum X^{2}-\left(\sum X\right)^{2} / N\right)\left(\sum Y^{2}-\left(\sum Y\right)^{2} / N\right)}}
$$

通过计算得到表 4 所示的实际响应曲线与理论 响应曲线的 Pearson 相关系数。

表 4 实际响应与理论响应的 Pearson 相关系数

\begin{tabular}{ccc}
\hline 控制方法 & 空载情况 & 带载情况 \\
\hline CGPI & 0.928 & 0.957 \\
DTPI & 0.938 & 0.979 \\
DTPISAKF & 0.991 & 0.993 \\
\hline
\end{tabular}

在带载的情况下, Pearson 相关系数的情况为 CGPI $<$ DTPI $<$ DTPISAKF，其中 DTPISAKF 的相关 系数达到了 0.993 的强匹配情形, 说明了在载荷惯 
量变化的情况下, DTPISAKF 能有效抵抗惯量摄动 的影响, 维持系统的设计性能, 验证了数字孪生驱 动复合控制策略的有效性。

\section{6 结论}

(1) 针对直驱部件在惯量摄动和装配特性变 化下的高精度伺服控制问题, 基于数字孪生框架提 出了模型参数同步方法和复合控制策略, 提出基于 性能指标约束的控制器和扩张状态卡尔曼滤波器设 计方法, 搭建直驱系统实验测试系统进行对比实验, 验证数字孪生驱动的复合控制策略的有效性。

（2）在变负载情况下，通过采集实际物理系统 的速度信息和控制器输出数据, 数字孪生模型快速 准确地检测到系统惯量 $J$ 由 $6.5 \times 10^{-3} \mathrm{~kg} \cdot \mathrm{m}^{2}$ 增加到 了 $8.9 \times 10^{-3} \mathrm{~kg} \cdot \mathrm{m}^{2}$, 其他装配特性参数变化很小, 这与伺服系统实际参数的变化过程相吻合。

（3）在速度正弦指令跟随精度实验中, 随着负 载惯量的变化, 数字孪生驱动的复合控制策略能够 保持系统稳定性和伺服性能, 速度残差均方根值相 对传统方法降低了 70\%。

（4）在速度阶跃指响应特性实验中，基于数字 孪生驱动的复合控制策略能够保持系统调整时间始 终为设计的 $0.1 \mathrm{~s}$, 阶跃响应曲线与理论响应曲线的 Pearson 相关系数达到了 0.993 , 验证了数字孪生驱 动的复合控制策略的有效性。

\section{参 考 文 献}

[1] FELIU V, PEREIRA E, DiAZ I M. Passivity-based control of single-link flexible manipulators using a linear strain feedback $[\mathrm{J}]$. Mechanism and Machine Theory, 2014, 71: 191-208.

[2] 丁有爽, 肖曦. 基于极点配置的永磁同步电机驱动柔性 负载 PI 调节器参数确定方法 $[\mathrm{J}]$. 中国电机工程学报,

2017, 37(04):1225-1239.

DING Youshuang, XIAO Xi. Parameter tuning methods based on pole placement for PI controllers of flexible loads driven by PMSM[J]. Proceedings of the CSEE, 2017, 37(4): 1225-1239.

[3] 李国涛, 李兵, 郭宏伟, 等. 桁架式可展开抓取机构展 开动力学建模及其自适应鲁棒控制[J]. 机械工程学报, 2020, 56(5): 39-46.

LI Guotao, LI Bing, GUO Hongwei, et al. Deployment dynamic modelling and adaptive robust control of truss-shaped deployable grasping mechanism[J]. Journal of Mechanical Engineering，2020， 56(5): 39-46.

[4] 张文辉, 刘文艺, 叶晓平, 等. 自由漂浮空间机械臂基 于神经网络的鲁棒自适应控制[J]. 机械工程学报, 2012, 48(21): 36-40.

ZHANG Wenhui, LIU Wenyi, YE Xiaoping, et al. Robust adaptive control for free-floating space manipulators based on neural network[J]. Journal of Mechanical Engineering, 2012, 48(21): 36-40.

[5] WANG Gang, LU Xiaoping, ZHAO Yunlong, et al. Neural network-based adaptive motion control for a mobile robot with unknown longitudinal slipping[J]. Chinese Journal of Mechanical Engineering, 2019, 32(1): 61

[6] HUANG Hai, ZHANG Guocheng, LI Jiyong, et al. Model based adaptive control and disturbance compensation for underwater vehicles[J]. Chinese Journal of Mechanical Engineering, 2018, 31(1): 19.

[7] TAO F, CHENG J, QI Q. Digital twin-driven product design, manufacturing and service with big data[J]. International Journal of Advanced Manufacturing Technology, 2018, 94(9-12): 3563-3576.

[8] CHEN J, YANG J, ZHOU H, et al. CPS Modeling of CNC machine tool work processes using an instruction-domain based approach[J]. Engineering, 2015, 1(2): 247-260.

[9] LIU C, CAO S, TSE W, et al. Augmented reality-assisted intelligent window for cyber-physical machine tools $[\mathrm{J}]$. Journal of Manufacturing Systems，2017，44: 280-286.

[10] 庞宇. 行星齿轮箱数字孪生体动力学仿真与故障诊断 研究[D]. 太原: 中北大学, 2020 .

PANG Yu. Simulation study on dynamics and fault diagnosis of digital twin in planetary gearbox[D]. Taiyuan: North University of China, 2020.

[11] PI S, LIU Q, LIU Q. A novel dynamic contour error estimation and control in high-speed $\mathrm{CNC}[\mathrm{J}]$. The International Journal of Advanced Manufacturing Technology, 2018, 96(1): 547-560.

[12] RIDWAN F, XU X. Advanced CNC system with in-process feed-rate optimisation[J]. Robotics and Computer-Integrated Manufacturing, 2013, 29(3): 12-20.

[13] TONG X, LIU Q, PI S, et al. Real-time machining data application and service based on IMT digital twin[J]. Journal of Intelligent Manufacturing, 2020, 31(5): 1113-1132.

[14] WANG X, WU Y, ZHANG E, et al. Characteristic 
model-based adaptive controller with discrete extended state observer for servo systems[J]. Proceedings of the Institution of Mechanical Engineers, Part I: Journal of Systems and Control Engineering, 2017, 231(4): 259-270.

[15] 郭飞燕, 刘检华, 邹方, 等. 数字孪生驱动的装配工艺 设计现状及关键实现技术研究 [J]. 机械工程学报, 2019, 55(17): 110-132.

GUO Feiyan, LIU Jianhua, ZOU Fang, et al. Research on the state-of-art, connotation and key implementation technology of assembly process planning with digital twin[J]. Journal of Mechanical Engineering, 2019, 55(17): 110-132.

[16] GRIEVES M. Product lifecycle management: The new paradigm for enłerprises[J]. International Journal of
Product Development, 2005, 2(1-2): 71-84.

作者简介: 江献良, 男, 1991 年出生, 博士研究生。主要研究方向为伺 服系统与数控。

E-mail: jx1123gfkd@163.com

范大鹏(通信作者), 男, 1964 年出生, 博士, 教授, 博士研究生导师。 主要研究方向为数控技术、嵌入式系统以及精密机电测控技术。

E-mail: fdp@nudt.edu.cn

陈凌宇, 男, 1990 年出生, 博士研究生。主要研究方向为伺服系统与 数控。

E-mail: 25873003@qq.com

郑杰基, 男, 1993 年出生, 博士研究生。主要研究方向为伺服系统与 数控。

E-mail: zhengjieji19@163.com

谭若愚, 男, 1990 年出生, 博士研究生。主要研究方向为精密电磁驱动 功能部件机电集成一体化设计。

E-mail: ruoyutan_nudt@qq.com

李宝宇, 男, 1991 年出生, 博士研究生。主要研究方向为智能装备精密 工程。

E-mail: libaoyu_jx2014@163.com 